\title{
Gezielte Manipulation des Darm-Mikrobioms derzeit nicht ratsam
}

\author{
Das Darm-Mikrobiom ist vermutlich an der Pathogenese vieler chronischer Erkrankungen betei- \\ ligt. Ergeben sich daraus bald auch Therapieansätze? Diabetologen äußerten sich beim DDG- \\ Kongress für ihr Fachgebiet zurückhaltend. Viele Fragen seien noch durch weitere Studien zu \\ klären, für klare Empfehlungen zum präventiven Einsatz etwa von Prä- und Probiotika sei es \\ noch zu früh.
}

Nach großen Fortschritten der Mikrobiom-Forscher im letzten Jahrzehnt und der Hoffnung auf ein neues Therapiekonzept durch gezielte Modifikation der Bakterienbesiedelung scheint jetzt wieder etwas Ruhe und vorsichtige Zurückhaltung einzukehren. Das Mikrobiom, das sich in den ersten zwei Lebensjahren manifestiert und erheblich von genetischen Faktoren abhängig ist, ist für schnelle Schlussfolgerungen zu komplex. „Die Bakterienstämme und -klassen, die unseren Darm besiedeln, können zwischen Diabetikern und Nichtdiabetikern variieren", konstatierte Dr. Volker Burkart, Immunbiologe an der Universität Düsseldorf. Aber das Mikrobiom ist eben auch relativ individuell, abhängig von genetischen Faktoren, Umwelt, Nahrung, Medikamenten und evtl. vielen weiteren Faktoren. „Es ist offen, welche Spezies optimal sind und was ein gesundes Mikrobiom ausmacht", sagte Burkart.

\section{Therapeutisches Potenzial vorhanden}

Das therapeutische Potenzial einer Modifikation des Darm-Mikrobioms wird weiterhin gesehen. Günstige Effekte sind zum Beispiel für eine gesunde Diät belegt. Bereits innerhalb von 24 Stunden kann dadurch die Zusammensetzung des Mikrobioms verändert und die Zahl der Bacteroides, der "guten Bakterien”, erhöht werden, berichtete Burkart. Weniger kohlenhydratreiche Getränke, mehr Früchte, mehr Joghurts wurden bei adipösen Patienten etwa mit einer größeren Vielfalt von Darmbakterien (Diversität) und geringeren inflammatorischen Markern assoziiert. Zugleich korreliert eine geringe Bakteriendiversität mit vermehrten Stoffwechselstörungen und subklinischen Entzündungsreaktionen. Auch Probiotika, vor allem Bifidobakterien und Laktobazillen, können das Darm-Mikrobiom positiv verändern. Zum Beispiel wurde bei Typ-2-Diabetikern durch Verzehr von L. acidophilus und B. lactis über 6 Wochen der Glukosemetabolismus verbessert (Ejtahed et al., Nutrition 2012; 28: 539) und bei glukosetoleranten Patienten durch L. reuteri die Inkretin- und Insulinsekretion erhöht (Simon et al., Diab Care 2015;38:1827), so Burkart. Ähnliche Zusammenhänge wurden auch für Präbiotika gefunden, etwa vermehrte Zufuhr von nicht verdaubaren Kohlenhydraten wie Oligofruktose und Inulin, die als Nahrungsgrundlage von „guten” Darmbakterien gelten.

Dennoch: Eine gezielte Aufnahme einzelner Bakterienstämme zur Diabetesprävention kann derzeit nicht empfohlen werden, sagte Burkart. Die Daten reichten dafür nicht aus. Und eine Stuhltransplantation von schlanken Spendern auf Menschen mit metabolischen Syndrom befinde sich zur Zeit im experi- mentellen Stadium, auch wenn es erste Hinweise gebe, dass dadurch die Insulin-Sensitivität verbessert werden könne.

\section{Bei Kindern mit Typ-1-Diabetes}

\section{Bakterienkommunikation beeinträchtigt}

Als „erst mal enttäuschend” bezeichnete Prof. Annette-Gabriele Ziegler vom Helmholtz-Zentrum München neue Daten zum möglichen Zusammenhang zwischen dem Mikrobiom bei Kindern und dem Auftreten von Typ-1-Diabetes. Die Daten der internationalen TEDDY-Studie bei rund 840 Kindern, darunter die Hälfte positiv auf Insel-Autoantikörper getestet, bestätigten die bereits bekannten Daten der BABYDIET-Studie mit 44 Kindern: Es kann nicht zwischen einer guten und einer bösen Bakterienbesiedelung unterschieden werden, betonte Ziegler, die Unterschiede zwischen beiden Gruppen seien minimal, auch bei der Bakteriendiversität. Einzige Auffälligkeit in beiden Studien: Auf struktureller Bakterienebene (Beta-Diversität), das heißt in den Netzwerken, der Stabilität und bei der Kommunikation der Mikroben, zeigten sich bei Kindern mit Insel-Autoantikörpern Beeinträchtigungen im Vergleich zu Gesunden. Das soll weiter erforscht werden.

Roland Fath

Symposium: „Das Darm-Mikrobiom in der Pathogenese des Diabetes mellitus", 51. DDG-Jahrestagung, 04.-07.05.2016, Berlin

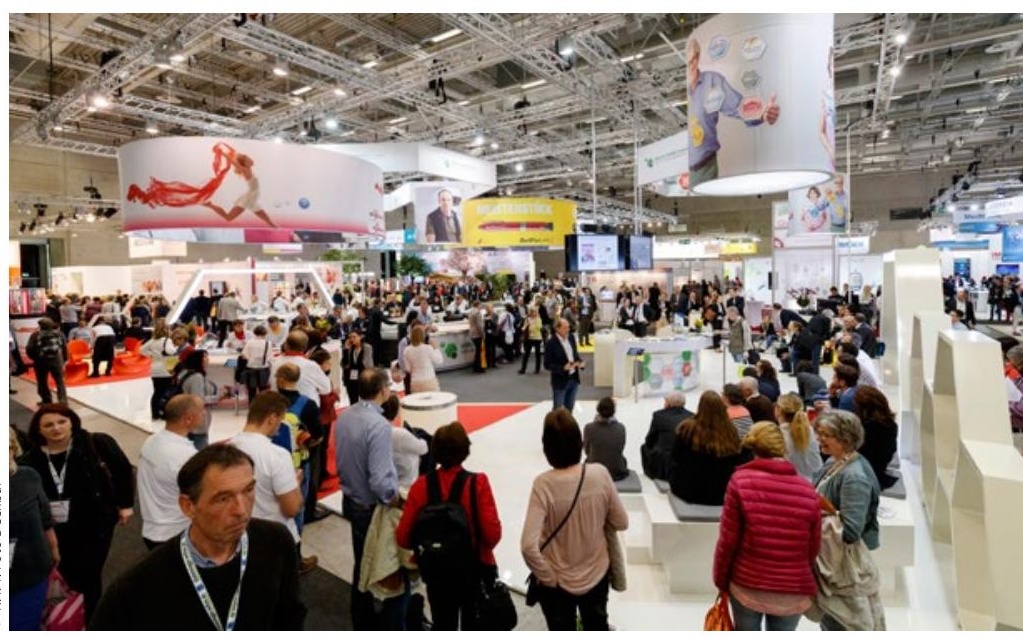

Auch die Besucher der Industrieausstellung beim DDG-Kongress unterscheiden sich hinsichtlich ihres Darm-Mikrobioms. 